\title{
Results of the first survey for the current status of inflammatory bowel disease management in Asian countries
}

\author{
Ji Won Kim \\ Department of Internal Medicine, Seoul National University College of Medicine, SMG-SNU Boramae Medical Center, Seoul, Korea
}

Article: Differences in the public medical insurance systems for inflammatory bowel disease treatment in Asian countries (Intest Res 2016;14:218-223)

Diagnosis of inflammatory bowel disease in Asia: the results of a multinational web-based survey in the $2^{\text {nd }}$ AOCC meeting in Seoul (Intest Res 2016;14:224-230)

Treatment of inflammatory bowel disease in Asia: the results of a multinational web-based survey in the $2^{\text {nd }}$ AOCC meeting in Seoul (Intest Res 2016;14:231-239)

Quality of care in inflammatory bowel disease in Asia: the results of a multinational web-based survey in the $2^{\text {nd }}$ AOCC meeting in Seoul (Intest Res 2016;14:240-247)

Although IBD was once considered a rare disorder in Asia compared with Western countries, the incidence and prevalence of IBD in Asia have both recently increased. Until now, many Asian physicians treating IBD patients have referred to consensus guidelines provided by Western committees. However, the characteristics of IBD patients differ geographically with respect to epidemiology, phenotype, and genetic susceptibility. The variability of IBD across different countries may alter the practices of different public health care systems, including health insurance coverage, greatly impacting patient outcome. Therefore, it is timely to survey many Asian countries for the current status of IBD patient care with respect to public medical insurance systems, diagnosis, treatment, and quality of care. ${ }^{1-4}$

This survey was originally planned by the organizing

Received May 30, 2016. Revised May 30, 2016.

Accepted May 30, 2016.

Correspondence to Ji Won Kim, Department of Internal Medicine, Seoul National University College of Medicine, SMG-SNU Boramae Medical Center, 20 Boramae-ro 5-gil, Dongjak-gu, Seoul 07061, Korea. Tel: +82-2-870-2221, Fax:+82-2-870-3863, E-mail: kjwjor@snu.ac.kr

Financial support: None. Conflict of interest: None. committee of the Asian Organization for Crohn's and Colitis (AOCC) and designed for one program of the $2^{\text {nd }}$ annual AOCC meeting, which was held in Seoul, Korea in June 2014. The aim of the survey was to identify how Asian physicians diagnose and treat their IBD patients. A survey to determine differences in public medical insurance systems and the quality of care across countries was also performed. This survey consisted of 4 parts including general information (9 items), diagnosis of IBD (18 items), treatment of IBD (30 items), and quality of IBD care (36 items). The survey was conducted through a web-based system between March 2014 and May 2014. The questionnaire was sent to medical doctors caring for IBD patients in Asia via e-mail by members of the organizing committee of the AOCC representing each Asian country. A total of 353 Asian medical doctors treating IBD patients responded to the survey (114 in China, 88 in Japan, 116 in Korea, 17 in Taiwan, 8 in Hong Kong, 4 in India, 3 in Singapore, and 1 each in the Philippines, Malaysia, and Indonesia).

In Western countries, IBD affected the personal lives of patients and caused substantial costs to health care systems. ${ }^{5}$ Wei et al. ${ }^{1}$ investigated the current status of public medical

๑ Copyright 2016. Korean Association for the Study of Intestinal Diseases. All rights reserved.

This is an Open Access article distributed under the terms of the Creative Commons Attribution Non-Commercial License (http://creativecommons.org/licenses/by-nc/4.0)

which permits unrestricted non-commercial use, distribution, and reproduction in any medium, provided the original work is properly cited. 
insurance systems among Asian countries using questionnaires about the costs and insurance coverage rate of costs for IBD, including diagnosis using endoscopy and treatment with 5-aminosalicylic acid (5-ASA) and biologics. The results of this survey showed that the public health insurance coverage rate of costs was high in Taiwan, Japan, South Korea, China, Hong Kong, and Singapore, but low in Malaysia and India. This difference affected use of expensive medications, especially biologics. The percentage of $\mathrm{CD}$ cases treated with biologics was $30 \%-40 \%$ in Japan, whereas the percentage was only $1 \%$ in India. Costs for conventional therapies, such as 5-ASA, steroids, and immunomodulators, were paid by public health insurance in Taiwan, Japan, China, Hong Kong, and Korea, whereas these costs were paid by patients in Singapore, Malaysia, and India. The fecal calprotectin test was available in clinical practice in Singapore and India, costing about US $\$ 40$ to US $\$ 80$. However, it was only available for research purposes in Taiwan, Japan, South Korea, and Malaysia. Tests for anti-tumor necrosis factor (TNF) agent serum levels and antibodies against anti-TNF agents were not yet available in all Asian countries.

Kim et al. ${ }^{2}$ analyzed how Asian physicians approached diagnosis of IBD. It is often difficult to accurately diagnose IBD in Asia due to the presence of various infectious diseases that mimic IBD, which may delay accurate diagnosis. For instance, intestinal tuberculosis, which is relatively prevalent in Asia, presents with similar symptoms as CD. The national diagnostic guidelines for IBD were commonly used in Korea, ${ }^{6,7}$ China, and Japan, ${ }^{8}$ but other Asian countries frequently used the "European Crohn's and Colitis Organisation" (ECCO) guidelines. For clinical assessment of disease activity of UC, most physicians used the Mayo Scoring System except Chinese physicians, who commonly used the Truelove and Witts severity index score. The CD activity index was overwhelmingly chosen for the clinical assessment of CD activity by physicians from all Asian countries. For small bowel evaluation in CD, there was a wide range of variation among countries. Physicians from Korea and China predominantly performed CT enterography, whereas those from Japan and other countries preferred small bowel follow through or balloon-assisted enteroscopy. For evaluation of perianal lesions, pelvic MRI was the most commonly used examination in all Asian countries. Across all Asian countries, over $20 \%$ of patients were given anti-tuberculosis treatment before CD diagnosis, reflecting the difficulty in discriminating between $\mathrm{CD}$ and intestinal tuberculosis, especially in the small bowel. ${ }^{9}$

Nakase et al. ${ }^{3}$ investigated IBD treatment protocols throughout Asia and showed that clinical management of
IBD patients varied among Asian countries. For example, the frequency of use of certain drugs for IBD treatment such as budesonide and tacrolimus differed among Asian countries, and induction therapies for mild to moderate inflammatory small bowel CD also differed. However, common therapeutic strategies for refractory IBD and active UC were employed throughout Asian countries. Treatment strategies for steroid-refractory acute severe UC were as follows: after initially using intravenous steroids for 5-7 days, most Asian physicians with the exception of those in Japan selected antiTNF agents, followed by cyclosporine.

Many Japanese physicians favored tacrolimus, followed by anti-TNF agents, while a few Japanese physicians selected apheresis therapies. This result suggests there may be differences in available medical treatments among Asian countries. More than half of physicians in Korea, China, and Japan always tested for cytomegalovirus infection in cases of severe UC. However, only $12 \%$ of other Asian physicians always performed this test. Treatment of severe inflammatory small bowel CD greatly differed when comparing Asian and Western countries. ${ }^{10}$ Only 3\% of Asian physicians selected budesonide, while most Asian physicians selected a combination of prednisolone and 5-ASA, followed by antiTNF agents, nutritional therapy, and thiopurines. Many Asian physicians chose thiopurines and anti-TNF agents for the treatment of steroid-dependent or refractory CD and UC patients.

There is a need to identify risk factors for poor prognosis in Asian CD patients to establish guidelines for early use of anti-TNF therapies. In Western countries, patients with risk factors such as age $<40$ years at diagnosis, presence of perianal lesions, early need for steroid, severe endoscopic lesions, and the existence of an NOD2/CARD15 mutation would be recommended for early treatment with anti-TNF agents (Top-down therapy). ${ }^{11}$ When patients become resistant to anti-TNF agent therapy, serum levels of anti-TNF agents or antibodies to infliximab or adalimumab are measured to determine subsequent therapeutic strategies such as increasing the dose of anti-TNF agents or changing to another antiTNF agent. ${ }^{12}$ However, most Asian physicians answered "No" when asked whether they monitored serum infliximab levels or antibodies to infliximab. Facilities for monitoring of these values were limited in Asian countries.

Song et al. ${ }^{4}$ evaluated the current status of quality of IBD care in Asian countries using a questionnaire-based survey developed by the American Gastroenterological Association entitled "an adult IBD physician performance measures set" that comprised 11 specific measures. ${ }^{13}$ The results showed 
that 7 of the 11 performance measures were executed well by more than $70 \%$ of physicians in Asian countries. Tuberculosis screening before anti-TNF therapy and documentation of IBD were consistently among the highest ranked measures, whereas pneumococcal immunization and prophylaxis of venous thromboembolisms (VTEs) in hospitalized patients were the lowest ranked in all countries. These lower ranked performance measures among Asian countries were comparable to a similarly low compliance in Western countries. ${ }^{14}$ In this survey, only $24 \%$ of Asian physicians performed well in considering prophylaxis for VTEs, likely due to limited data about the risk of VTEs in Asian IBD patients leading physicians to consider this practice unimportant.

Awareness of performance measures differed among Asian countries. For example, $72.4 \%$ of Korean vs. $39.8 \%$ of Japanese physicians reported awareness of pneumococcal immunization, while $60 \%$ of Korean vs. $48.9 \%$ of Japanese physicians reported awareness of influenza immunization. Reasons for non-performance varied among countries, especially with regard to lower ranked performance measures. For instance, pneumococcal and influenza immunizations were considered unimportant in most Asian countries, whereas lack of time was the main reason these immunizations were not performed in Korea. This survey had limitations since relying on the memories of the physicians may not accurately reflect actual performance in each country.

These 4 articles report the results of the first survey to assess the public medical insurance system, diagnosis, treatment, and quality of care for IBD in multiple Asian countries. There is currently no guideline for the diagnosis and treatment of IBD in Asian patients. Therefore, many Asian physicians commonly use guidelines of Western countries, such as the ECCO guidelines. These articles in the current issue of Intestinal Research may be the cornerstone to establishing specific guidelines for the improved management of IBD patients in Asian countries.

\section{REFERENCES}

1. Wei SC. Differences in the public medical insurance systems for inflammatory bowel disease treatment in Asian countries. Intest Res 2016;14:218-223.
2. Kim ES, Chen M, Lee J, Lee CK, Kim YS. Diagnosis of inflammatory bowel disease in Asia: the results of a multinational webbased survey in the $2^{\text {nd }}$ Asian Organization for Crohn's and Colitis (AOCC) meeting in Seoul. Intest Res 2016;14:224-230.

3. Nakase H, Keum B, Ye BD, Park SJ, Koo HS, Eun CS. Treatment of inflammatory bowel disease in Asia: the results of a multinational web-based survey in the $2^{\text {nd }}$ Asian Organization of Crohn's and Colitis (AOCC) meeting in Seoul. Intest Res 2016;14:231-239.

4. Song HK, Lee KM, Jung SA, Hong SN, Han DS, Yang SK; IBD study group of Korean Association for the Study of Intestinal Diseases (KASID). Quality of care in inflammatory bowel disease in Asia: the results of a multinational web-based survey in the $2^{\text {nd }}$ Asian Organization of Crohn's and Colitis (AOCC) meeting in Seoul. Intest Res 2016;14:240-247.

5. Burisch J, Jess T, Martinato M, Lakatos PL; ECCO -EpiCom. The burden of inflammatory bowel disease in Europe. J Crohns Colitis 2013;7:322-337.

6. Ye BD, Jang BI, Jeen YT, et al. Diagnostic guideline of Crohn's disease. Korean J Gastroenterol 2009;53:161-176.

7. Choi CH, Jung SA, Lee BI, et al. Diagnostic guideline of ulcerative colitis. Korean J Gastroenterol 2009;53:145-160.

8. Ueno F, Matsui T, Matsumoto T, et al. Evidence-based clinical practice guidelines for Crohn's disease, integrated with formal consensus of experts in Japan. J Gastroenterol 2013;48:31-72.

9. Kim SH, Kim JW, Jeong JB, Lee KL, Kim BG, Choi YH. Differential diagnosis of Crohn's disease and intestinal tuberculosis in patients with spontaneous small-bowel perforation. Dig Surg 2014;31:151-156.

10. Bernstein CN. Treatment of IBD: where we are and where we are going. Am J Gastroenterol 2015;110:114-126.

11. Dubinsky MC. Serologic and laboratory markers in prediction of the disease course in inflammatory bowel disease. World J Gastroenterol 2010;16:2604-2608.

12. Guerra I, Bermejo F. Management of inflammatory bowel disease in poor responders to infliximab. Clin Exp Gastroenterol 2014;7:359-367.

13. Melmed GY, Siegel CA, Spiegel BM, et al. Quality indicators for inflammatory bowel disease: development of process and outcome measures. Inflamm Bowel Dis 2013;19:662-668.

14. Sam JJ, Bernstein CN, Razik R, Thanabalan R, Nguyen GC. Physicians' perceptions of risks and practices in venous thromboembolism prophylaxis in inflammatory bowel disease. Dig Dis Sci 2013;58:46-52. 